\title{
The Solubility-Permeability Interplay in Using Cyclodextrins as Pharmaceutical Solubilizers: Mechanistic Modeling and Application to Progesterone
}

\author{
ARIK DAHAN, ${ }^{1}$ JONATHAN M. MILLER, ${ }^{1}$ AMNON HOFFMAN, ${ }^{2}$ GREGORY E. AMIDON, ${ }^{1}$ GORDON L. AMIDON ${ }^{1}$ \\ ${ }^{1}$ Department of Pharmaceutical Sciences, College of Pharmacy, University of Michigan, Ann Arbor, Michigan $48109-1065$ \\ ${ }^{2}$ Department of Pharmaceutics, School of Pharmacy, Faculty of Medicine, The Hebrew University of Jerusalem, Israel
}

Received 4 August 2009; revised 23 September 2009; accepted 31 October 2009

Published online 28 December 2009 in Wiley InterScience (www.interscience.wiley.com). DOI 10.1002/jps.22033

\begin{abstract}
A quasi-equilibrium mass transport analysis has been developed to quantitatively explain the solubility-permeability interplay that exists when using cyclodextrins as pharmaceutical solubilizers. The model considers the effects of cyclodextrins on the membrane permeability $\left(P_{\mathrm{m}}\right)$ as well as the unstirred water layer (UWL) permeability $\left(P_{\mathrm{aq}}\right)$, to predict the overall effective permeability $\left(P_{\text {eff }}\right)$ dependence on cyclodextrin concentration $\left(C_{\mathrm{CD}}\right)$. The analysis reveals that: (1) UWL permeability markedly increases with increasing $C_{\mathrm{CD}}$ since the effective UWL thickness quickly decreases with increasing $C_{\mathrm{CD}}$; (2) membrane permeability decreases with increasing $C_{\mathrm{CD}}$, as a result of the decrease in the free fraction of drug; and (3) since $P_{\text {aq }}$ increases and $P_{\mathrm{m}}$ decreases with increasing $C_{\mathrm{CD}}$, the UWL is effectively eliminated and the overall $P_{\text {eff }}$ tends toward membrane control, that is, $P_{\text {eff }} \approx P_{\mathrm{m}}$ above a critical $C_{\mathrm{CD}}$. Application of this transport model enabled excellent quantitative prediction of progesterone $P_{\text {eff }}$ as a function of HP $\beta C D$ concentrations in PAMPA assay, Caco-2 transepithelial studies, and in situ rat jejunal-perfusion model. This work demonstrates that when using cyclodextrins as pharmaceutical solubilizers, a trade-off exists between solubility increase and permeability decrease that must not be overlooked; the transport model presented here can aid in striking the appropriate solubility-permeability balance in order to achieve optimal overall absorption. (C) 2009 Wiley-Liss, Inc. and the American Pharmacists Association J Pharm Sci 99:2739-2749, 2010
\end{abstract}

Keywords: low-solubility drugs; cyclodextrins; solubility-permeability interplay; drug transport analysis; intestinal absorption

\section{INTRODUCTION}

Modern drug discovery techniques (e.g., advances in high throughput screening methods, the introduction of combinatorial chemistry) have resulted in an increase in the number of drug candidates being selected that exhibit low solubility in water. By some estimates, more than $40 \%$ of new drug candidates are lipophilic and have poor aqueous solubility. ${ }^{1-3}$ With very minor exceptions, dissolution of the drug substance in the aqueous gastrointestinal (GI) milieu is a prerequisite for absorption following oral administration. Hence, compounds with inadequate aqueous solubility often suffer from limited oral bioavailability. A great challenge facing the pharma-

Arik Dahan and Jonathan M. Miller contributed equally to this work.

Correspondence to: Gordon L. Amidon (Telephone: 734-764-2226; Fax: 734-763-6282; E-mail: glamidon@umich.edu)

Journal of Pharmaceutical Sciences, Vol. 99, 2739-2749 (2010) (c) 2009 Wiley-Liss, Inc. and the American Pharmacists Association ceutical scientist is to formulate these molecules into orally administered dosage forms with sufficient bioavailability. ${ }^{4,5}$

Among the various approaches to improve the aqueous solubility of lipophilic drugs, the utilization of cyclodextrins has become widespread in recent years. ${ }^{6,7}$ Cyclodextrins are crystalline, nonhygroscopic, cyclic oligosaccharides, with a hydrophilic outer surface and a less hydrophilic central cavity which is able to host hydrophobic solutes. From a drug delivery standpoint, cyclodextrins have gained extensive attention and use, due to their ability to increase the solubility of hydrophobic drugs via the formation of more water soluble inclusion complexes. 8,9

According to the Biopharmaceutics Classification System (BCS), ${ }^{10}$ the extent of oral absorption of a drug is governed by two primary factors: (1) the effective permeability across the intestinal mucosa and (2) the solubility and dissolution characteristics in the GI milieu. ${ }^{11-13}$ While increased solubility of a lipophilic drug can be achieved by a cyclodextrins 
based delivery system, the effect of such a formulation on the apparent permeability is not completely understood. Several studies have shown that the use of cyclodextrins can reduce the apparent permeability of the drug, ${ }^{14-16}$ an effect that is often qualitatively attributed to a decrease in the free fraction of the drug available for membrane permeation. These opposing effects of cyclodextrins on solubility and permeability can lead to paradoxical effects on the overall fraction of drug absorbed. Indeed, a critical review of the literature reveals that the use of cyclodextrins can result in enhanced, unchanged, or even decreased oral bioavailability. ${ }^{17,18} \mathrm{As}$ a result of these observations, qualitative guidelines for the proper use of cyclodextrins have been proposed, ${ }^{14,18,19}$ however no quantitative analysis that enables simulation of the overall effect of cyclodextrins on intestinal membrane permeability is currently available.

The aims of this research were to develop mathematical mass transport models to explain the impact of molecular complexation with cyclodextrins on intestinal membrane permeation, and to mechanistically elucidate the interplay between the opposing effects of cyclodextrins on apparent solubility and permeability. To evaluate the mathematical theory, the models were applied to the highly lipophilic, lowsolubility, BCS class II drug progesterone,${ }^{20}$ utilizing several in vitro and in situ intestinal membrane transport models, that is, PAMPA, Caco-2 cell monolayers, and single-pass rat jejunal perfusion. Overall, this work provides an increased understanding of the underlying mechanisms that govern the effects of molecular complexation on intestinal membrane transport, and enables the more efficient and intelligent use of molecular complexation strategies to facilitate oral absorption.

\section{THEORY}

\section{Quasi-Equilibrium Analysis of the Effect of Cyclodextrins on Membrane Transport}

The intrinsic membrane permeability of free drug $\left(P_{\mathrm{m}(F)}\right)$ in the absence of cyclodextrins can be written as: ${ }^{21}$

$$
P_{\mathrm{m}(F)}=\frac{D_{\mathrm{m}(F)} K_{\mathrm{m}(F)}}{h_{\mathrm{m}(F)}}
$$

where $D_{\mathrm{m}(F)}$ is the membrane diffusion coefficient of the free drug in the absence of cyclodextrins, $K_{\mathrm{m}(F)}$ the membrane/aqueous partition coefficient of drug in the absence of cyclodextrins, and $h_{\mathrm{m}(F)}$ the membrane thickness experienced by free drug in the absence of cyclodextrins.
Likewise, the apparent membrane permeability of the drug in the presence of cyclodextrins $\left(P_{\mathrm{m}}\right)$ can be written as:

$$
P_{\mathrm{m}}=\frac{D_{\mathrm{m}} K_{\mathrm{m}}}{h_{\mathrm{m}}}
$$

where $D_{\mathrm{m}}$ is the apparent membrane diffusion coefficient of the drug in the presence of cyclodextrins and $K_{\mathrm{m}}$ is the apparent membrane/aqueous partition coefficient of the drug in the presence of cyclodextrins.

Assuming only the free drug permeates the membrane such that $D_{\mathrm{m}(F)}=D_{\mathrm{m}}$ and that the presence of cyclodextrins does not effect the membrane thickness such that $h_{\mathrm{m}(F)}=h_{\mathrm{m}}$, Eqs. (1) and (2) can be combined to give:

$$
P_{\mathrm{m}}=\frac{P_{\mathrm{m}(F)} K_{\mathrm{m}}}{K_{\mathrm{m}(F)}}
$$

$K_{\mathrm{m}(F)}$ and $K_{\mathrm{m}}$ can be expressed as:

$$
\begin{gathered}
K_{\mathrm{m}(F)}=\frac{S_{\mathrm{m}(F)}}{S_{\mathrm{aq}(F)}} \\
K_{\mathrm{m}}=\frac{S_{\mathrm{m}}}{S_{\mathrm{aq}}}
\end{gathered}
$$

where $S_{\mathrm{m}(F)}$ is the membrane solubility of the free drug, $S_{\text {aq }(F)}$ the aqueous solubility of the free drug, $S_{\mathrm{m}}$ the apparent membrane solubility of the drug in the presence of cyclodextrins, and $S_{\mathrm{aq}}$ the apparent aqueous solubility of the drug in the presence of cyclodextrins.

Assuming that the presence of cyclodextrins does not affect the drug solubility in the membrane such that $S_{\mathrm{m}(F)}=S_{\mathrm{m}}$, Eqs. (3)-(5) can be combined to give:

$$
P_{\mathrm{m}}=\frac{P_{\mathrm{m}(F)} S_{\mathrm{aq}(F)}}{S_{\mathrm{aq}}}
$$

Assuming 1:1 complexation between drug and cyclodextrins, the dependence of drug solubility on cyclodextrins concentration $\left(C_{\mathrm{CD}}\right)$ can be written as:

$$
S_{\mathrm{aq}}=S_{\mathrm{aq}(F)}\left(K_{11 \mathrm{aq}} C_{\mathrm{CD}}+1\right)
$$

where $K_{11 \mathrm{aq}}$ is the aqueous association constant of the 1:1 drug/cyclodextrins complex.

Eqs. (6) and (7) can be combined to express the $P_{\mathrm{m}}$ dependence on $C_{\mathrm{CD}}$ :

$$
P_{\mathrm{m}}=\frac{P_{\mathrm{m}(F)}}{\left(K_{11 \mathrm{aq}} C_{\mathrm{CD}}+1\right)}
$$

Recognizing that the fraction of free drug $(F)$ can be written as:

$$
F=\frac{S_{\mathrm{aq}(F)}}{S_{\mathrm{aq}}}=\frac{1}{K_{11 \mathrm{aq}} C_{\mathrm{CD}}+1}
$$


Eqs. (8) and (9) can be combined to express the $P_{\mathrm{m}}$ dependence on $F$ :

$$
P_{\mathrm{m}}=P_{\mathrm{m}(F)} F
$$

The intrinsic permeability of the free drug through the unstirred aqueous boundary layer $\left(P_{\mathrm{aq}(F)}\right)$ in the absence of cyclodextrins can be written as: ${ }^{22}$

$$
P_{\mathrm{aq}(F)}=\frac{D_{\mathrm{aq}(F)}}{h_{\mathrm{aq}(F)}}
$$

where $D_{\mathrm{aq}(F)}$ is the diffusion coefficient of the free drug through the unstirred aqueous boundary layer in the absence of cyclodextrins and $h_{\mathrm{aq}(F)}$ is the unstirred aqueous boundary layer thickness experienced by free drug in the absence of cyclodextrins.

Likewise, the apparent unstirred aqueous boundary layer permeability of the drug in the presence of cyclodextrins $\left(P_{\mathrm{aq}}\right)$ can be written as:

$$
P_{\mathrm{aq}}=\frac{D_{\mathrm{aq}}}{h_{\mathrm{aq}}}
$$

where $D_{\text {aq }}$ is the apparent diffusion coefficient of the drug through the unstirred aqueous boundary layer in the presence of cyclodextrins and $h_{\mathrm{aq}}$ is the apparent unstirred aqueous boundary layer thickness in the presence of cyclodextrins.

Eqs. (11) and (12) can be combined to give:

$$
P_{\mathrm{aq}}=\frac{P_{\mathrm{aq}(F)} D_{\mathrm{aq}} h_{\mathrm{aq}(F)}}{D_{\mathrm{aq}(F)} h_{\mathrm{aq}}}
$$

$D_{\text {aq }}$ may be expressed as: ${ }^{22}$

$$
D_{\text {aq }}=F D_{\text {aq }(F)}+B D_{\text {aq }(B)}
$$

where $B$ is the fraction of drug molecules bound to cyclodextrins $(B=1-F)$ and $D_{\mathrm{aq}(B)}$ is the aqueous diffusion coefficient of the drug-cyclodextrin complex. Since the molecular size of the cyclodextrin is much larger than that of the free drug, $D_{\mathrm{aq}(B)}$ may be assumed to be approximately equal to the aqueous diffusion coefficient of free cyclodextrin, $D_{\text {aq(CD) }}$ :

$$
D_{\mathrm{aq}(B)} \approx D_{\mathrm{aq}(\mathrm{CD})}
$$

Likewise, $h_{\text {aq }}$ may be expressed as:

$$
h_{\mathrm{aq}}=F h_{\mathrm{aq}(F)}+B h_{\mathrm{aq}(B)}
$$

where $h_{\mathrm{aq}(B)}$ is the apparent unstirred aqueous boundary layer thickness experienced by the drugcyclodextrin complex.

Eqs. (11), (13), (14), and (16) can be combined to show the dependence of $P_{\mathrm{aq}}$ on both free and bound drug:

$$
P_{\mathrm{aq}}=\frac{\left(F D_{\mathrm{aq}(F)}+B D_{\mathrm{aq}(B)}\right)}{\left(F h_{\mathrm{aq}(F)}+B h_{\mathrm{aq}(B)}\right)}
$$

Assuming that the unstirred aqueous boundary layer is no longer relevant for the drug-cyclodextrin complex (i.e., $h_{\mathrm{aq}(B)}=0$ and $h_{\mathrm{aq}}=F h_{\mathrm{aq}(F)}$ ), Eqs. (9) and (17) can be combined to express the $P_{\text {aq }}$ dependence on $C_{\mathrm{CD}}$ :

$$
P_{\mathrm{aq}}=\frac{P_{\mathrm{aq}(F)} D_{\mathrm{aq}}\left(K_{11 \mathrm{aq}} C_{\mathrm{CD}}+1\right)}{D_{\mathrm{aq}(F)}}
$$

where the dependence of $D_{\mathrm{aq}}$ on $C_{\mathrm{CD}}$ may be calculated using Eqs. (14) and (15).

Taking into account the membrane permeability as well as the unstirred aqueous boundary layer permeability on either side of the membrane, $P_{\mathrm{aq}(1)}$ and $P_{\mathrm{aq}(2)}$, the overall effective permeability $\left(P_{\text {eff }}\right)$ of the drug can be written as: ${ }^{22}$

$$
P_{\text {eff }}=\frac{1}{1 / P_{\mathrm{aq}(1)}+1 / P_{\mathrm{m}}+1 / P_{\mathrm{aq}(2)}}
$$

For simplicity, $P_{\mathrm{aq}(2)}$ is assumed to have a negligible effect, such that $P_{\mathrm{aq}(1)}=P_{\mathrm{aq}}$, and Eq. (19) can be rewritten as:

$$
P_{\text {eff }}=\frac{1}{1 / P_{\text {aq }}+1 / P_{\mathrm{m}}}
$$

Thus, the overall $P_{\text {eff }}$ dependence on $C_{\mathrm{CD}}$ may be predicted via Eq. (20) wherein the $P_{\mathrm{m}}$ and $P_{\mathrm{aq}}$ dependence on $C_{\mathrm{CD}}$ are predicted using Eqs. (8) and (18) with knowledge of $P_{\mathrm{m}(F)}, K_{11 \text { aq }}, P_{\mathrm{aq}(F)}, D_{\mathrm{aq}(F)}$, and $D_{\text {aq(CD) }}$.

Moreover, the drug concentration at the interface between the UWL and membrane $\left(C_{\text {aq Membrane Surface }}\right)$ as a function of $C_{\mathrm{CD}}$ can be calculated according to the following equation: ${ }^{22}$

$$
\frac{C_{\text {aqMembraneSurface }}}{C_{\text {aqBulk }}}=\frac{P_{\text {eff }}}{P_{\mathrm{m}}}
$$

The assumptions nested in these analyses include: (1) quasi-equilibrium conditions (2) 1:1 stoichiometry between drug/cyclodextrin complex; (3) only the free drug partitions and diffuses into the membrane, but not the drug/cyclodextrin complex, such that $S_{\mathrm{m}(F)}=S_{\mathrm{m}}$ and $D_{\mathrm{m}(F)}=D_{\mathrm{m}} ;$ (4) the presence of cyclodextrins does not affect the membrane thickness such that $h_{\mathrm{m}(F)}=h_{\mathrm{m}}$; and (5) only the free drug encounters a significant unstirred aqueous boundary layer thickness but the unstirred aqueous boundary layer thickness is negligible for the drugcyclodextrins complex such that $h_{\mathrm{aq}(B)}=0$.

\section{MATERIALS AND METHODS}

\section{Materials}

Progesterone, 2-hydroxypropyl- $\beta$-cyclodextrins (HP $\beta$ $\mathrm{CD})$, phenol red and hexadecane were purchased from 
Sigma Chemical Co. (St. Louis, MO). Hexane, potassium chloride, and $\mathrm{NaCl}$ were obtained from Fisher Scientific, Inc. (Pittsburgh, PA). Acetonitrile and water (Acros Organics, Geel, Belgium) were HPLC grade. All other chemicals were of analytical reagent grade.

\section{Solubility Studies}

The solubility experiments for progesterone with HP $\beta C D$ were conducted according to the method described by Higuchi and Connors. ${ }^{23}$ To a number of test tubes containing excess amounts of progesterone, $0-0.015 \mathrm{M}$ aqueous HP $\beta C D$ solutions were added. The intrinsic solubility in water was determined from five individual samples without HP $\beta C D$. The test tubes were tightly closed and placed in a shaking water bath at $25^{\circ} \mathrm{C}$ and $100 \mathrm{rpm}$. Establishment of equilibrium was assured by comparison of samples after 24 and $48 \mathrm{~h}$. Before sampling, the vials were centrifuged at $10,000 \mathrm{rpm}$ for $10 \mathrm{~min}$. Supernatant was carefully withdrawn from each test tube, filtered, and immediately assayed for drug content by HPLC.

\section{Caco-2 Cell Monolayer Assay}

Caco-2 cells (passage 25-32) from American Type Culture Collection (Rockville, MD) were routinely maintained in Dulbecco's modified Eagle's medium (DMEM, Invitrogen Corp., Carlsbad, CA) containing $10 \%$ fetal bovine serum, $1 \%$ nonessential amino acids, $1 \mathrm{mM}$ sodium pyruvate, and $1 \%$ L-glutamine. Cells were grown in an atmosphere of $5 \% \mathrm{CO}_{2}$ and $90 \%$ relative humidity at $37^{\circ} \mathrm{C}$. The DMEM medium was routinely replaced by fresh medium every 3 days. Cells were passaged upon reaching approximately 80\% confluence using $4 \mathrm{~mL}$ trypsin-EDTA (Invitrogen Corp.).

Transepithelial transport studies were performed using a method described previously. ${ }^{24,25}$ Briefly, $5 \times 10^{4}$ cells $/ \mathrm{cm}^{2}$ were seeded onto collagen-coated membranes (12-well Transwell plate, $0.4-\mu \mathrm{m}$ pore size, $12 \mathrm{~mm}$ diameter, Corning Costar, Cambridge, MA) and were allowed to grow for 21 days. Mannitol and Lucifer yellow permeabilities were assayed for each batch of Caco-2 monolayers $(n=3)$, and TEER measurements were performed on all monolayers (Millicell-ERS epithelial Voltohmmeter, Millipore Co., Bedford, MA). Monolayers with apparent mannitol and Lucifer yellow permeability $<3 \times 10^{-7} \mathrm{~cm} / \mathrm{s}$, and TEER values $>300 \Omega \mathrm{cm}^{2}$ were used for the study. On the day of the experiment, the DMEM was removed and the monolayers were rinsed and incubated for $20 \mathrm{~min}$ with a blank transport buffer. The apical transport buffer contained $1 \mathrm{mM} \mathrm{CaCl}$, $0.5 \mathrm{mM} \mathrm{MgCl}_{2} \cdot 6 \mathrm{H}_{2} \mathrm{O}, 145 \mathrm{mM} \mathrm{NaCl}, 3 \mathrm{mM} \mathrm{KCl}, 1 \mathrm{mM}$ $\mathrm{NaH}_{2} \mathrm{PO}_{4}, 5 \mathrm{mM}$ D-glucose, and $5 \mathrm{mM}$ MES, at pH 6 . Following the $20 \mathrm{~min}$ incubation, the drug free transport buffer was removed from the apical side and replaced by $0.5 \mathrm{~mL}$ of progesterone solution in the uptake buffer, with or without HP $\beta C D$. Throughout the experiment, the transport plates were kept in a shaking incubator $(50 \mathrm{rpm})$ at $37^{\circ} \mathrm{C}$ (unless stated otherwise). Samples were taken from the receiver (basolateral) side at various time points up to $120 \mathrm{~min}$ $(50 \mu \mathrm{L})$, and similar volumes of blank buffer were added following each sample withdrawal. At the last time point $(120 \mathrm{~min})$, sample was taken from the donor (apical) side as well, in order to confirm mass balance. Samples were immediately assayed for drug content. Caco-2 monolayers were checked for confluence by measuring the TEER before and after the transport study.

Permeability coefficient $\left(P_{\text {app }}\right)$ across Caco-2 cell monolayers was calculated from the linear plot of drug accumulated in the receiver side versus time, using the following equation:

$$
P_{\text {app }}=\frac{1}{C_{0} A} \times \frac{\mathrm{d} Q}{\mathrm{~d} t}
$$

where $\mathrm{d} Q / \mathrm{d} t$ is the steady-state appearance rate of the drug on the receiver side, $C_{0}$ the initial concentration of the drug in the donor side, and $A$ the monolayer growth surface area $\left(1.12 \mathrm{~cm}^{2}\right)$. Linear regression was carried out to obtain the steady-state appearance rate of the drug on the receiver side.

\section{Parallel Artificial Membrane Permeation Assay (PAMPA)}

PAMPA studies were carried out using a method described previously with minor modifications. ${ }^{26,27}$ Solutions of progesterone $(25 \mu \mathrm{M})$ were prepared with $\mathrm{HP} \beta C D$ at concentrations of $0,0.1,0.2,0.4,0.8$, and $1.6 \mathrm{mM}$ in phosphate buffer saline (PBS) $\mathrm{pH}$ 7.4. PAMPA experiments were carried out in Millipore (Danvers, MA) 96-well MultiScreen-Permeability filter plates with $0.3 \mathrm{~cm}^{2}$ polycarbonate filter support $(0.45 \mu \mathrm{m})$. The filter supports in each well were first impregnated with $15 \mu \mathrm{L}$ of a $5 \%$ solution $(\mathrm{v} / \mathrm{v})$ of hexadecane in hexanes. The wells were then allowed to dry for $1 \mathrm{~h}$ to ensure complete evaporation of the hexanes resulting in a uniform layer of hexadecane. The donor wells were then loaded with $0.15 \mathrm{~mL}$ of the progesterone-HP $\beta C D$ solution and each receiver well was loaded with $0.3 \mathrm{~mL}$ of PBS. Five wells were loaded at each HP $\beta C D$ concentration to enable collection of a well at times points of $30,60,90$, 120 , and $150 \mathrm{~min}$ and each experiment was repeated three times for a total of 15 wells per HP $\beta C D$ concentration. The donor plate was then placed upon the 96-well receiver plate and the resulting PAMPA sandwich was incubated at $25^{\circ} \mathrm{C}$. Receiver plate wells ( $n=3$ for each HP $\beta C D$ concentration) were then collected every $30 \mathrm{~min}$ over $2.5 \mathrm{~h}$ and the progesterone concentration in each well was determined by HPLC. Permeability coefficient $\left(P_{\text {app }}\right)$ across Caco-2 cell monolayers was calculated from the linear plot of 
drug accumulated in the receiver side versus time using Eq. (22).

\section{Rat Jejunal Perfusion}

All animal experiments were conducted using protocols approved by the University Committee of Use and Care of Animals (UCUCA), University of Michigan, and the animals were housed and handled according to the University of Michigan Unit for Laboratory Animal Medicine guidelines. Male albino Wistar rats (Charles River, IN) weighing 250-300 g were used for all perfusion studies. Prior to each experiment, the rats were fasted overnight (12-18 h) with free access to water. Animals were randomly assigned to the different experimental groups.

The procedure for the in situ single-pass intestinal perfusion followed previously published reports. ${ }^{28,29}$ Briefly, rats were anesthetized with an intramuscular injection of $1 \mathrm{~mL} / \mathrm{kg}$ of ketamine-xylazine solution (9\%:1\%, respectively) and placed on a heated surface maintained at $37^{\circ} \mathrm{C}$ (Harvard Apparatus Inc., Holliston, MA). The abdomen was opened by a midline incision of 3-4 cm. A $10 \mathrm{~cm}$ proximal jejunal segment was carefully exposed and cannulated on two ends with flexible PVC tubing $(2.29 \mathrm{~mm}$ i.d., inlet tube $40 \mathrm{~cm}$, outlet tube $20 \mathrm{~cm}$, Fisher Scientific, Inc.). Care was taken to avoid disturbance of the circulatory system, and the exposed segment was kept moist with $37^{\circ} \mathrm{C}$ normal saline solution. Solutions of progesterone $(25 \mu \mathrm{M})$ were prepared with $\mathrm{HP} \beta \mathrm{CD}$ at concentrations of $0,0.025,0.25$, and $2.5 \mathrm{mM}$ in the perfusate buffer. This buffer consisted of $10 \mathrm{mM}$ MES buffer, $\mathrm{pH}$ $6.5,135 \mathrm{mM} \mathrm{NaCl}, 5 \mathrm{mM} \mathrm{KCl}$, and $0.1 \mathrm{mg} / \mathrm{mL}$ phenol red, a nonabsorbable marker for measuring water flux. All perfusate solutions were incubated in a $37^{\circ} \mathrm{C}$ water bath. The isolated segment was first rinsed with blank perfusion buffer at a flow rate of $0.5 \mathrm{~mL} /$ min to clean out any residual debris. At the start of the study, the test solutions were perfused through the intestinal segment (Watson Marlow Pumps 323S, Watson-Marlow Bredel, Inc., Wilmington, MA) at a flow rate of $0.2 \mathrm{~mL} / \mathrm{min}$. The perfusion buffer was first perfused for $1 \mathrm{~h}$, in order to ensure steady state conditions (as also assessed by the inlet over outlet concentration ratio of phenol red which approaches 1 at steady state). After reaching steady state, samples were taken in $10 \mathrm{~min}$ intervals for $1 \mathrm{~h}$. All samples, including perfusion samples at different time points, original drug solution, and inlet solution taken at the exit of the syringe were immediately assayed by HPLC. Following the termination of the experiment, the length of each perfused jejunal segment was accurately measured.

The net water flux in the single-pass rat jejunal perfusion studies, resulting from water absorption in the intestinal segment, was determined by measurement of phenol red, a nonabsorbed, nonmetabolized marker. The measured $C_{\text {out }} / C_{\text {in }}$ ratio was corrected for water transport according to the following equation:

$$
\frac{C_{\text {out }}^{\prime}}{C_{\text {in }}^{\prime}}=\frac{C_{\text {out }}}{C_{\text {in }}} \times \frac{C_{\text {in phenol red }}}{C_{\text {out phenol red }}}
$$

where $C_{\text {in phenol red }}$ is the concentration of phenol red in the inlet sample, and $C_{\text {out }}$ phenol red is the concentration of phenol red in the outlet sample. The effective permeability $\left(P_{\text {eff }}\right)$ through the rat gut wall in the single-pass intestinal perfusion studies was determined assuming the "plug flow" model expressed in the following equation: ${ }^{30}$

$$
P_{\text {eff }}=\frac{-Q \ln \left(C_{\text {out }}^{\prime} / C_{\text {in }}^{\prime}\right)}{2 \pi R L}
$$

where $Q$ is the perfusion buffer flow rate, $C^{\prime}{ }_{\text {out }} / C^{\prime}$ in is the ratio of the outlet concentration and the inlet concentration of the tested drug that has been adjusted for water transport via Eq. (23), $R$ is the radius of the intestinal segment (set to $0.2 \mathrm{~cm}$ ), and $L$ is the length of the intestinal segment.

\section{High-Performance Liquid Chromatography (HPLC)}

HPLC analyses were performed on an Agilent Technologies (Palo Alto, CA) HPLC 1100 equipped with photodiode array detector and ChemStation for LC 3D software. Progesterone was assayed using an Agilent Technologies $150 \mathrm{~mm} \times 4.6 \mathrm{~mm} \mathrm{XDB}^{-\mathrm{C}_{18}}$ column with $5 \mu \mathrm{m}$ particle size. The detection wavelength was $242 \mathrm{~nm}$. The mobile phase consisted of $30: 70(\mathrm{v} / \mathrm{v}) 0.1 \%$ trifluoroacetic acid in water: $0.1 \%$ trifluoroacetic acid in acetonitrile and was pumped at a flow rate of $1.0 \mathrm{~mL} / \mathrm{min}$. Injection volumes for all HPLC analyses ranged from 5 to $100 \mu \mathrm{L}$. Separate standard curves were used for each experiment $\left(R^{2}>0.99\right)$.

\section{Statistical Analysis}

All in vitro experiments were performed in triplicate (unless stated otherwise), and all animal experiments were $n=4$. Values are expressed as the means \pm the standard deviation (SD). To determine statistically significant differences among the experimental groups, the nonparametric Kruskal-Wallis test was used for multiple comparisons, and the two-tailed nonparametric Mann-Whitney $U$-test for two-group comparison where appropriate. A $p$-value of less than 0.05 was termed significant.

\section{RESULTS}

\section{Effect of HPßCD on Progesterone Solubility}

The solubility data for complex formation between progesterone and HP $\beta C D$ is presented in Figure 1. Progesterone solubility increased linearly $\left(R^{2}>0.99\right)$ with increasing HP $\beta C D$ concentrations as per Eq. (7). 


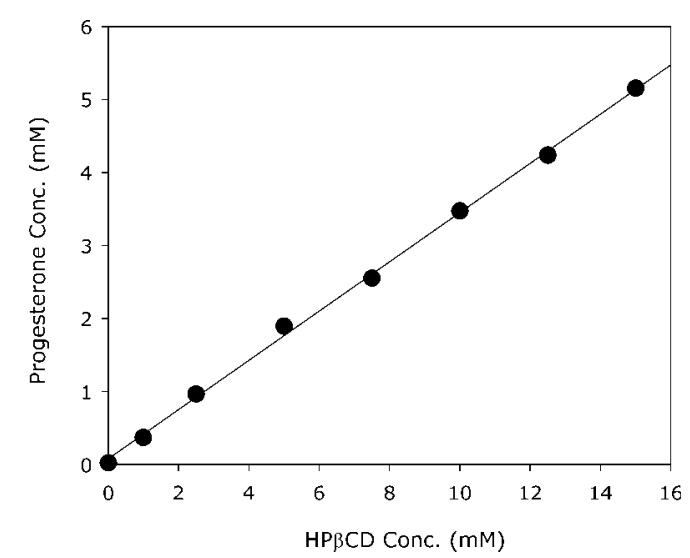

Figure 1. Aqueous solubility of progesterone as a function of increasing $\mathrm{HP} \beta \mathrm{CD}$ concentration at $25^{\circ} \mathrm{C}$. Data are presented as means $\pm \mathrm{SD}$ (error bars smaller than symbols); $n=3$ in each experimental group.

The linearity of the phase solubility diagram indicates 1:1 stoichiometric complexation between progesterone and $\mathrm{HP} \beta C D$. A very strong binding constant of $14,324 \mathrm{M}^{-1}$ was calculated from the solubility data via Eq. (7).

\section{Effect of HPBCD on Progesterone Transport Across Caco-2 Cell Monolayers}

The dependence of progesterone flux across Caco-2 cell monolayer, and the corresponding $P_{\text {app }}$ values, on $\mathrm{HP} \beta \mathrm{CD}$ concentration $(0$ and $75 \mu \mathrm{M})$ is presented in Figure 2. It can be seen that progesterone permeability decreased twofold in the presence of $75 \mu \mathrm{M}$ $\mathrm{HP} \beta \mathrm{CD}$ as compared to progesterone alone. These data were obtained under a constant rotation speed of
$50 \mathrm{rpm}$. To determine whether the results obtained under this condition represent the true membrane permeability $\left(P_{\mathrm{m}(F)}\right)$ of progesterone, that is, to assess the effect of the unstirred water layer (UWL), progesterone Caco-2 permeability was evaluated as a function of rotation speed (Fig. 3a). Significantly higher transport was evident with increased rotation speed from 0 , to 20 , and to $50 \mathrm{rpm}$, indicating that progesterone $P_{\text {app }}$ is limited by the UWL, at low rotation speeds. Additional increase in rotation speed to $70 \mathrm{rpm}$ failed to produce increased flux, indicating that the UWL does not limit the overall $P_{\text {app }}$ at high rotation speeds. Hence, it is evident that the $P_{\text {app }}$ value obtained at $50 \mathrm{rpm}\left(4.8 \times 10^{-5} \mathrm{~cm} / \mathrm{s}\right)$ approximates the true intrinsic membrane permeability $\left(P_{\mathrm{m}(F)}\right)$ of progesterone across Caco-2 monolayers.

The effect of rotation speed on progesterone Caco-2 membrane transport was also evaluated in the presence of $75 \mu \mathrm{M}$ HPßCD. As shown in Figure $3 b$, similar progesterone permeabilities were obtained at different rotation speeds $0-50 \mathrm{rpm}$, consistent with the idea that the UWL is no longer rate limiting and progesterone $P_{\text {app }}$ is under membrane control at an $\mathrm{HP} \beta \mathrm{CD}$ concentration as low as $75 \mu \mathrm{M}$. This observation is in corroboration with previous reports that cyclodextrins may reduce the UWL effect on the $P_{\text {app }}$ of lipophilic compounds. $8,16,31,32$

Figure 4 compares the predicted permeability of progesterone across Caco-2 cell monolayers as a function of $\mathrm{HP} \beta \mathrm{CD}$ concentration to the experimentally observed $P_{\text {app }}$ values. The theoretical lines were calculated via Eq. (8) $\left(P_{\mathrm{m}}\right)$, Eq. (18) $\left(P_{\mathrm{aq}}\right)$, and Eq. (20) $\left(P_{\text {eff }}\right)$ using the experimental values of $P_{\mathrm{m}(F)}=4.8 \times 10^{-5} \mathrm{~cm} / \mathrm{s}, K_{11 \mathrm{aq}}=14,324 \mathrm{M}^{-1}$,
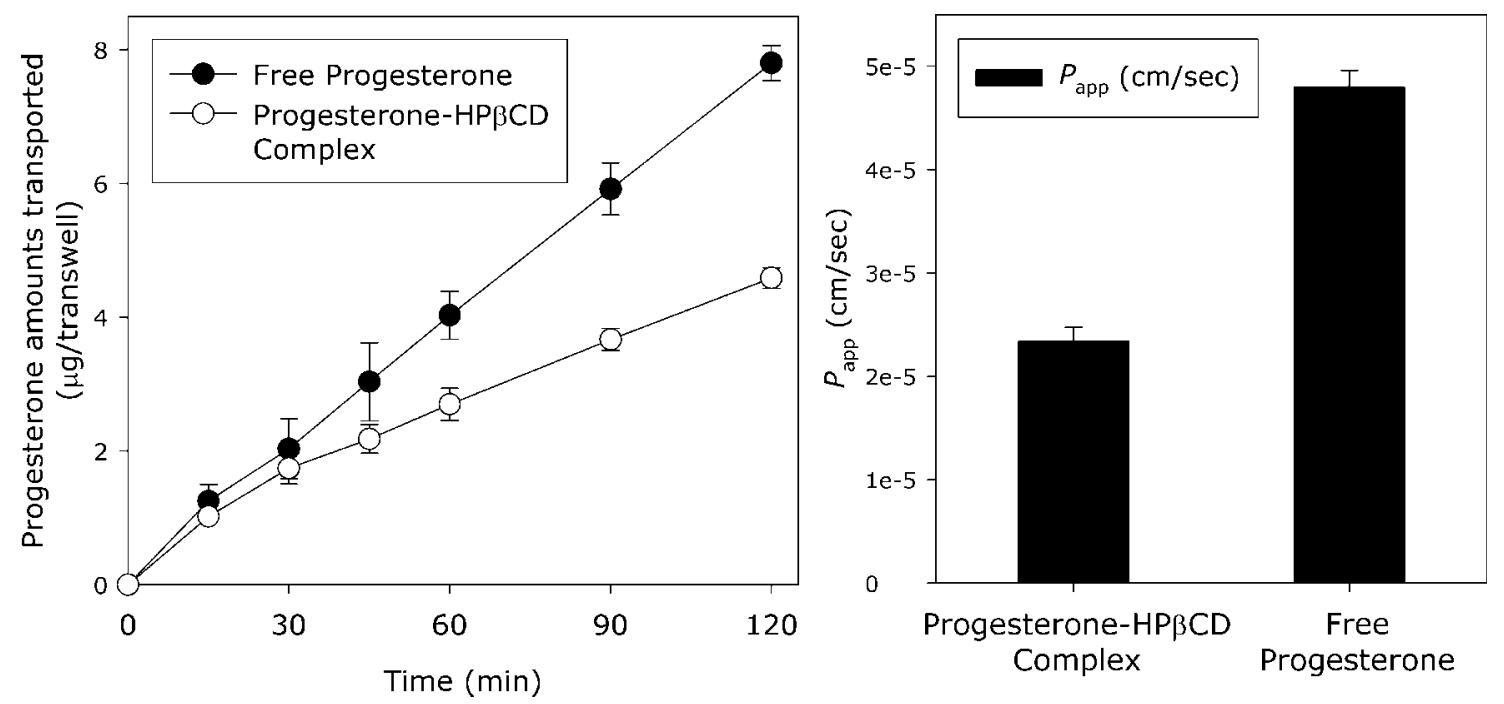

Figure 2. Progesterone $(25 \mu \mathrm{M})$ flux across Caco-2 monolayers alone $(\mathbf{O})$ and in the presence of $75 \mu \mathrm{M} H \mathrm{HP} \beta \mathrm{CD}(\bigcirc)$ at $50 \mathrm{rpm}$ rotation speed (left), and the corresponding $P_{\text {app }}$ values (right). Data are presented as means $\pm \mathrm{SD} ; n=3$ in each experimental group. 
a

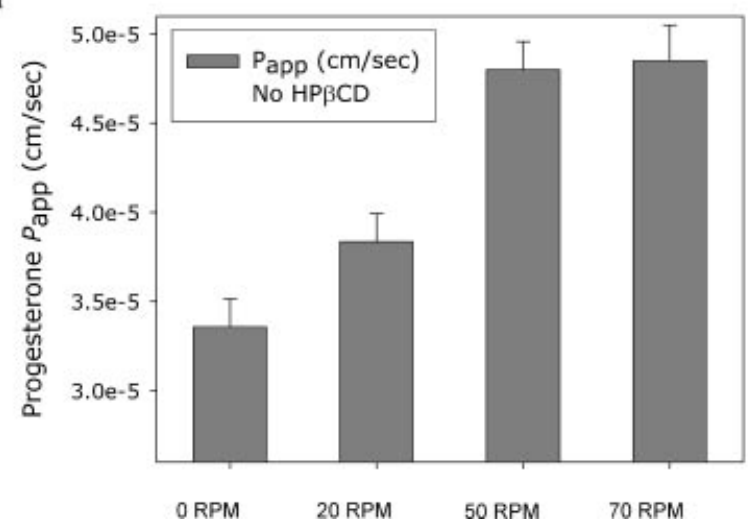

b

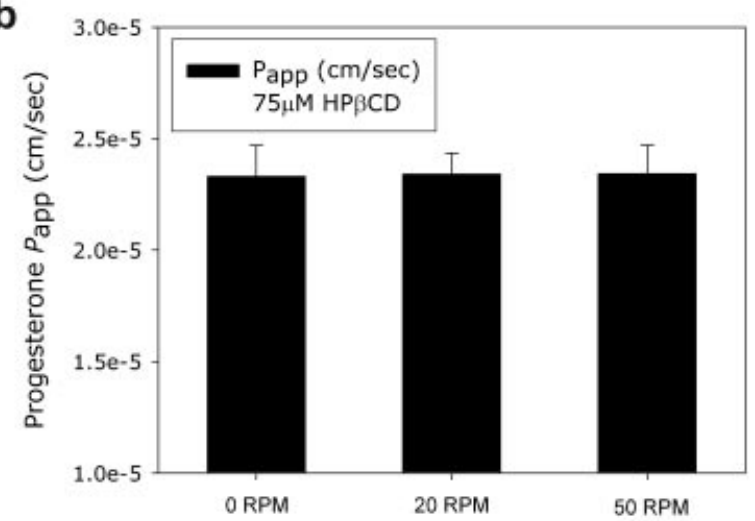

Figure 3. $P_{\text {app }}$ values of progesterone $(25 \mu \mathrm{M})$ across Caco- 2 monolayers at different rotation speeds, in the absence of HP $\beta C D$ (left; panel a) and in the presence of $75 \mu \mathrm{M}$ HP $\beta C D$ (right; panel b). Data are presented as means $\pm \mathrm{SD} ; n=3$ in each experimental group.

$D_{\text {aq }(F)}=8.5 \times 10^{-6} \mathrm{~cm}^{2} / \mathrm{s}$ from Amidon et al., ${ }^{22}$ and $D_{\mathrm{aq}(\mathrm{CD})}=3.2 \times 10^{-6} \mathrm{~cm}^{2} / \mathrm{s}$ from Ribeiro et al. ${ }^{33}$ The value of $P_{\mathrm{aq}(F)}$ was calculated to be $10.6 \times 10^{-5} \mathrm{~cm} / \mathrm{s}$ via Eq. (20) using the experimental $P_{\text {app }}$ value of $3.3 \times 10^{-5} \mathrm{~cm} / \mathrm{s}$ at $0 \mathrm{rpm}$ rotation speed in the absence of cyclodextrins for $P_{\text {eff }}$, and the experimental $P_{\text {app }}$ value of $4.8 \times 10^{-5} \mathrm{~cm} / \mathrm{s}$ at $50 \mathrm{rpm}$ rotation speed in the absence of cyclodextrins for $P_{\mathrm{m}(F)}$. The predicted values for both $P_{\text {eff }}$ and $P_{\mathrm{m}}$ agreed well with the experimentally observed $P_{\text {app }}$ value of $2.3 \times 10^{-5} \mathrm{~cm} / \mathrm{s}$ at a cyclodextrins concentration of $75 \mu \mathrm{M}$ (Fig. 4). In fact, the theoretical value of $P_{\mathrm{m}}$ calculated via Eq. (8) exactly matched the experimentally observed $P_{\text {app }}$ value.

\section{Effect of HP $\beta C D$ on Progesterone Transport in PAMPA Model}

The theoretical and experimental dependence of progesterone $P_{\text {app }}$ on HP $\beta C D$ concentration in the

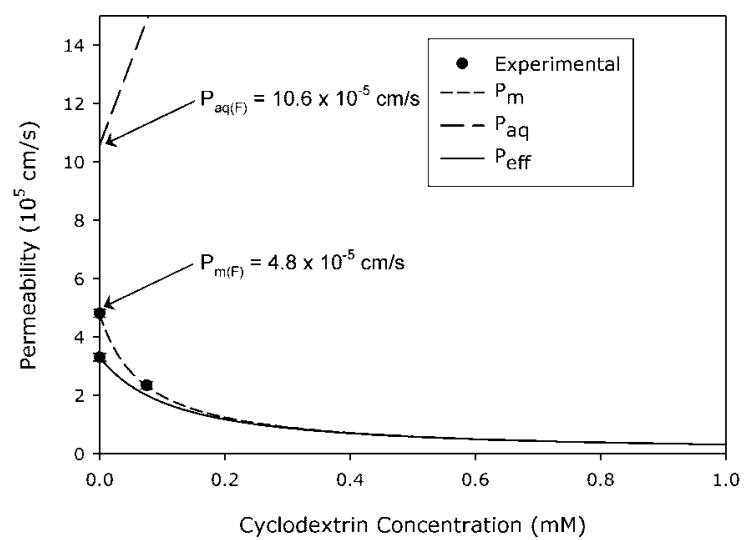

Figure 4. Permeability of progesterone across Caco-2 cell monolayers as a function of HP $\beta C D$ concentration. The theoretical lines were calculated via Eq. (8) $\left(P_{\mathrm{m}}\right)$, Eq. (18) $\left(P_{\mathrm{aq}}\right)$, and Eq. $(20)\left(P_{\text {eff }}\right)$. Experimental data points are presented as means $\pm \mathrm{SD} ; n=3$ in each experimental group. hexadecane-based PAMPA model is shown in Figure 5. Progesterone PAMPA permeability in the absence of HP $\beta C D$ was investigated at increasing rotation speeds as well, in order to assess the effect of the UWL (Fig. 6). Progesterone permeability decreased 2.1, 3.5, 6.4, 15.4, and 33.1 times at HP $\beta C D$ concentrations of $0.1,0.2,0.4,0.8$, and $1.6 \mathrm{mM}$, respectively, as compared to progesterone alone (Fig. 5). It can be seen that progesterone permeability in the PAMPA model remained constant with increasing rotation speed up to $100 \mathrm{rpm}$ (Fig. 6). This indicates that progesterone permeability is under membrane control in the hexadecane membrane PAMPA model. Thus, the effect of the UWL does not need to be considered and Eq. (8) may be directly applied to predict the dependence of progesterone apparent membrane permeability on $\mathrm{HP} \beta \mathrm{CD}$ concentration. Hence, the theoretical progesterone

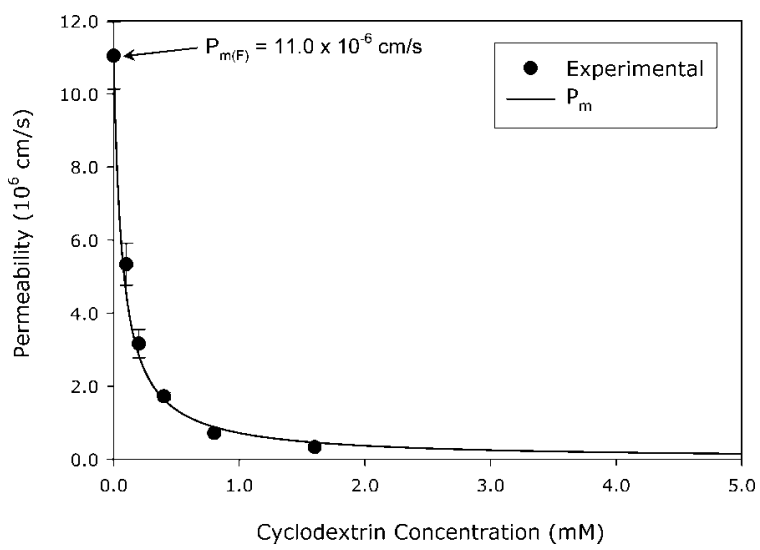

Figure 5. $P_{\text {app }}$ of progesterone as a function of $\mathrm{HP} \beta \mathrm{CD}$ concentration in the PAMPA assay. The theoretical line was calculated via Eq. (8) using the experimental values of $P_{\mathrm{m}}=11.0 \times 10^{-6} \mathrm{~cm} / \mathrm{s}$ and $K_{11 \mathrm{aq}}=14,324 \mathrm{M}^{-1}$. Experimental data points are presented as means $\pm \mathrm{SD} ; n=3$ in each experimental group. 


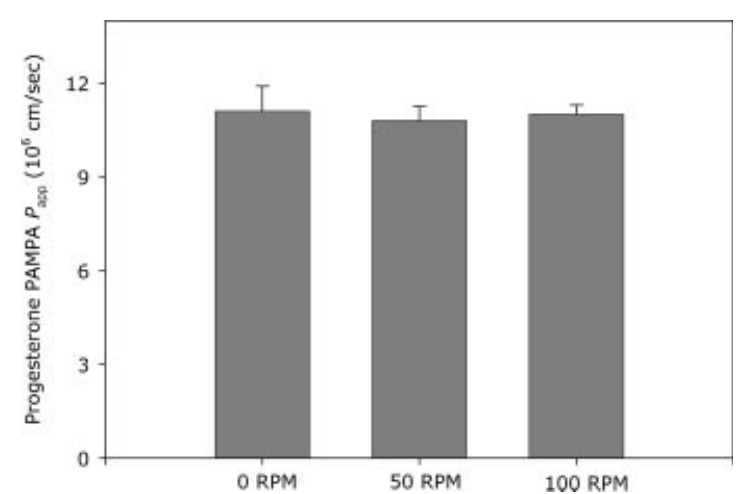

Figure 6. $P_{\text {app }}$ of progesterone $(25 \mu \mathrm{M})$ in the PAMPA model at different rotation speeds $(0,50$, and $100 \mathrm{rpm})$. Data are presented as means $\pm \mathrm{SD} ; n=3$ in each experimental group.

PAMPA permeability as a function of HP $\beta C D$ concentration illustrated in Figure 5 was calculated via Eq. (8) using the experimental values of $P_{\mathrm{m}(F)}=11 \times 10^{-6} \mathrm{~cm} / \mathrm{s}$ and $K_{11 \mathrm{aq}}=14,324 \mathrm{M}^{-1}$. Excellent agreement between the experimental data and the predicted values was obtained at all of the HP $\beta C D$ concentrations tested (Fig. 5).

\section{Effect of HP $\beta C D$ on Progesterone Rat Jejunal Permeability}

The theoretical and experimental progesterone permeability in the single-pass intestinal-perfusion rat model with increasing $\mathrm{HP} \beta \mathrm{CD}$ concentrations $(0$, $0.025,0.25$, and $2.5 \mathrm{mM}$ ) is illustrated in Figure 7. In the absence of $\mathrm{HP} \beta \mathrm{CD}$, progesterone showed very high permeability $\left(82.4 \times 10^{-5} \mathrm{~cm} / \mathrm{s}\right)$ across rat jejunum. The addition of an equi-molar $(25 \mu \mathrm{M})$ amount of $\mathrm{HP} \beta C D$ resulted in no significant increase in $P_{\text {eff }}$

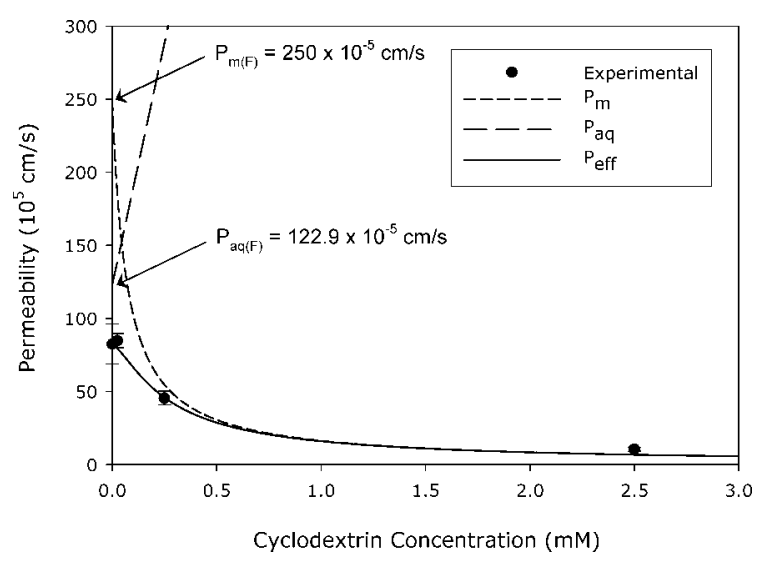

Figure 7. The effective permeability of progesterone as a function of HP $\beta C D$ concentration in the in situ rat jejunal perfusion model. The theoretical lines were calculated via Eq. (8) $\left(P_{\mathrm{m}}\right)$, Eq. (18) $\left(P_{\mathrm{aq}}\right)$, and Eq. $(20)\left(P_{\text {eff }}\right)$. Experimental data points are presented as means $\pm \mathrm{SD} ; n=4$ in each experimental group. $\left(84.8 \times 10^{-5} \mathrm{~cm} / \mathrm{s}\right)$. However, the $P_{\text {eff }}$ of progesterone decreased 1.8-fold in the presence of $0.25 \mathrm{mM} \mathrm{HP} \beta \mathrm{CD}$ and 7.8-fold in the presence of $2.5 \mathrm{mM}$ HP $\beta C D$. The predicted lines for $P_{\mathrm{m}}, P_{\mathrm{aq}}$, and $P_{\text {eff }}$ in Figure 7 were calculated via Eqs. (8), (18) and (20), respectively. The experimental parameters used in the calculations were $K_{11 \mathrm{aq}}=14,324 \mathrm{M}^{-1}$ (from this work), $D_{\mathrm{aq}(F)}=8.5 \times 10^{-6} \mathrm{~cm}^{2} / \mathrm{s},{ }^{22}$ and $D_{\mathrm{aq}(\mathrm{CD})}=3.2 \times$ $10^{-6} \mathrm{~cm}^{2} / \mathrm{s}{ }^{33}$ The experimental value for progesterone rat jejunal $P_{\mathrm{m}(F)}$ of $250 \times 10^{-5} \mathrm{~cm} / \mathrm{s}$ used in the calculations was previously estimated by determining the $P_{\text {eff }}$ of progesterone at increasing perfusate flow rates. ${ }^{34}$ The value of $P_{\mathrm{aq}(F)}$ used in the predictions was calculated according to Eq. (20) from the experimental values of $P_{\mathrm{m}(\mathrm{F})}$ and the $P_{\text {eff }}$ of progesterone determined in the absence of cyclodextrins $\left(82.4 \times 10^{-5} \mathrm{~cm} / \mathrm{s}\right)$. Excellent agreement was obtained between the experimental and predicted progesterone $P_{\text {eff }}$ values at all of the HP $\beta C D$ concentrations tested.

Figure 8 contains the predicted $D_{\text {aq }}, h_{\text {aq }}$, and $P_{\text {aq }}$ as a function of $\mathrm{HP} \beta C D$ concentration for progesterone in the rat jejunal perfusion model. The theoretical curves were calculated according to Eqs. (14)-(18). The value of $h_{\mathrm{aq}(F)}$ was calculated to be $103 \mu \mathrm{m}$ via Eq. (12) using the $P_{\text {eff }}$ of progesterone determined in the absence of cyclodextrins $\left(82.4 \times 10^{-5} \mathrm{~cm} / \mathrm{s}\right)$ and the progesterone $D_{\mathrm{aq}(F)}=8.5 \times 10^{-6} \mathrm{~cm}^{2} / \mathrm{s}$ from Amidon et al. ${ }^{22}$ Figure 9 shows the theoretical membrane surface to bulk concentration ratio $\left(C_{\mathrm{aq}}\right.$ Membrane Surface $/ C_{\text {aq Bulk }}$ ) of progesterone as a function of HP $\beta C D$ concentration in the rat jejunal perfusion model. The predicted curve was calculated via Eq. (21). Figure 10 illustrates the effect of HP $\beta C D$ on progesterone aqueous solubility and permeability based on the theoretical quasi-equilibrium transport analysis developed in this work. The apparent solubility and permeability as a function of HP $\beta C D$

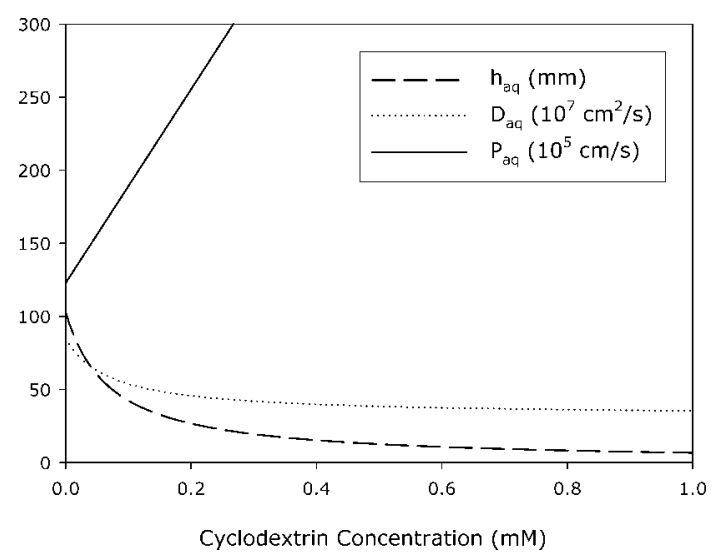

Figure 8. Dependence of $D_{\text {aq }}, h_{\text {aq }}$, and $P_{\text {aq }}$ on HP $\beta C D$ concentration for progesterone in the rat jejunal perfusion model. The theoretical lines were calculated according to Eqs. (14)-(18). 


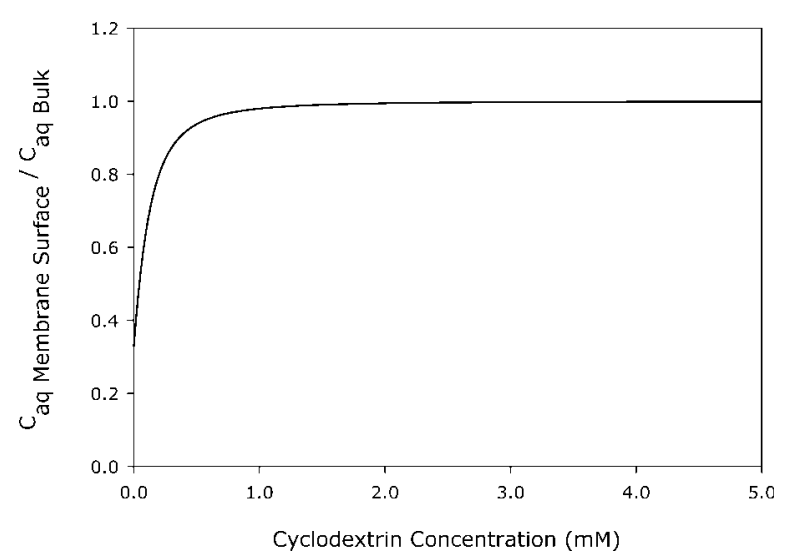

Figure 9. Theoretical membrane surface to bulk concentration ratio $\left(C_{\mathrm{aq} \text { Membrane Surface }} C_{\mathrm{aq} \text { Bulk }}\right)$ of progesterone as a function of $\mathrm{HP} \beta \mathrm{CD}$ concentration in the rat jejunal perfusion model, calculated according to Eq. (21).

concentration curves were calculated using Eqs. (7) and (20), respectively.

\section{DISCUSSION}

The trade-off between the apparent solubility increase and permeability decrease when using cyclodextrins as pharmaceutical solubilizers is described in this article. These opposing effects of cyclodextrin can lead to paradoxical effects on the overall fraction of drug absorbed. In this work, we offer a quantitative modeling of the interplay between the opposing effects of cyclodextrins on the apparent solubility and permeability, and show the excellent prediction of overall fraction of drug absorbed as a function of $C_{\mathrm{CD}}$ obtained by the model.

The very strong molecular complexation between progesterone and $\mathrm{HP} \beta C D$, as evidenced by the

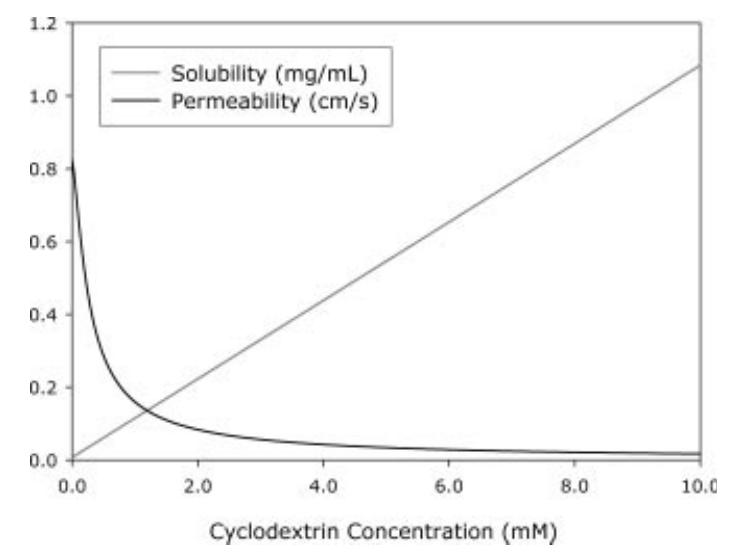

Figure 10. The effect of HP $\beta C D$ on progesterone solubility (gray) and permeability (black) based on the theoretical quasi-equilibrium transport analysis. Solubility and permeability were calculated using Eqs. (7) and (20), respectively. extremely high binding constant of $14,324 \mathrm{M}^{-1}$, results in substantially enhanced aqueous solubility (Fig. 1). Indeed, this solubility advantage provided by cyclodextrins is the primary reason for their widespread popularity and use. The high binding constant also results in very little free progesterone, even at relatively low HP $\beta C D$ concentrations. For example, the free fraction of progesterone is 0.5 at an $\mathrm{HP} \beta \mathrm{CD}$ concentration of only $70 \mu \mathrm{M}$, and at $\mathrm{HP} \beta \mathrm{CD}$ concentrations of $>1.4 \mathrm{mM}$, the free fraction of progesterone is $<0.05$. This decreased fraction of free drug can significantly alter membrane permeation behavior as was observed in this work using the PAMPA, Caco-2 cell monolayers, and in situ rat jejunal perfusion models.

In the Caco-2 studies, progesterone permeability decreased twofold in the presence of $75 \mu \mathrm{M}$ HP $\beta C D$ (Figs. 2 and 3), as compared to progesterone alone. In the absence of cyclodextrin, significantly higher permeabilities were observed with increased rotation speed from 0 , to 20 , and to $50 \mathrm{rpm}$, indicating that progesterone $P_{\text {app }}$ is limited by the UWL at low rotation speeds (Fig. 3a). This observation is in corroboration with previous reports. ${ }^{22,34-36}$ An additional increase in rotation speed to $70 \mathrm{rpm}$ failed to produce increased flux, indicating that in this experimental method, the UWL does not limit the overall $P_{\text {app }}$ at rotation speeds of $50 \mathrm{rpm}$ and above (Fig. 3a). Hence, it is evident that the $P_{\text {app }}$ value obtained at $50 \mathrm{rpm}\left(4.8 \times 10^{-5} \mathrm{~cm} / \mathrm{s}\right)$ approximates the true membrane permeability $\left(P_{\mathrm{m}(\mathrm{F})}\right)$ of progesterone across Caco-2 monolayers. In the presence of cyclodextrins, progesterone permeability was no longer limited by the UWL, as evidenced by the lack of rotation speed effect (Fig. 3b). This is in agreement with previous reports showing that cyclodextrins may reduce the UWL effect on the $P_{\text {app }}$ of lipophilic compounds. ${ }^{8,16,31,32}$ Since progesterone Caco-2 permeability is under membrane control at an HP $\beta C D$ concentration as low as $75 \mu \mathrm{M}$ and $50 \mathrm{rpm}$ stir speed, it follows that the predicted $P_{\mathrm{m}}$ (Eq. 8) exactly matches the experimentally observed $P_{\text {app }}$ value at $75 \mu \mathrm{M}$ HPßCD (Fig. 4).

Progesterone permeability was also significantly impacted by the presence of HP $\beta C D$ in the hexadecane-based PAMPA model (Fig. 5). The overall transport was unaffected by rotation speed (Fig. 6), indicating that in this experimental model progesterone permeability is not significantly effected by the UWL, rather the rate of transport across the hexadecane membrane is rate limiting. This is not to say that the UWL is not present in the PAMPA model, rather the effect of the UWL on overall permeability is insignificant as compared to the hexadecane membrane. Hence, the $P_{\text {app }}$ in the absence of $\mathrm{HP} \beta C D$ can be assumed to be approximately equal to $P_{\mathrm{m}(\mathrm{F})}$ and only the effect of HP $\beta C D$ on 
$P_{\mathrm{m}}$ (Eq. 8) needs to be considered (i.e., $P_{\text {eff }} \approx P_{\mathrm{m}}$ ). Indeed, excellent agreement was achieved between the experimental and predicted permeability of progesterone as a function of HP $\beta C D$ concentration in the PAMPA model when Eq. (8) was used for the predictions (Fig. 5).

Progesterone permeability across rat jejunal segments was also significantly altered by the presence of cyclodextrins. As shown in Figure 7, the progesterone $P_{\text {eff }}$ across the rat jejunum stayed relatively consistent at very low HP $\beta C D$ concentrations $(0.025 \mathrm{mM})$, but then decreased rapidly with increasing cyclodextrins concentration $(0.25-2.5 \mathrm{mM})$. This results because at very low cyclodextrins concentrations, the overall $P_{\text {eff }}$ is limited by the UWL. This is in corroboration with previous reports that have shown progesterone permeability to be limited by the aqueous boundary layer in the rat intestinal perfusion model. ${ }^{34,35}$ As shown in Figures 9 and 10, the predicted $P_{\text {aq }}$ increases markedly with increasing cyclodextrins concentration. This may be explained by the high value of $K_{11 \text { aq }}$, as the UWL is only experienced by the free drug and is effectively eliminated for the progesterone-HP $\beta C D$ complex (i.e., $h_{\mathrm{aq}(B)}=0$ ), such that the effective thickness of the boundary layer decreases with decreasing free fraction (i.e., $h_{\mathrm{aq}}=F h_{\mathrm{aq}(F)}$ ). This is in agreement with and explains some previous reports showing that cyclodextrins may reduce the UWL effect on the apparent permeability of lipophilic compounds. ${ }^{8,16,31,32}$ Figure 8 shows the predicted $D_{\text {aq }}$, $h_{\text {aq }}$, and $P_{\text {aq }}$ dependence on HP $\beta C D$ concentration, calculated via Eqs. (14)-(18). The value of progesterone $D_{\text {aq }}$ decreases with increasing cyclodextrins concentration, which does contribute to a slight decrease in the overall value of $P_{\text {aq }}$ as expressed in Eqs. (12) and (18). However, this effect is essentially negligible in comparison to the effect of the shrinking $h_{\mathrm{aq}}$, which leads to a marked increase in $P_{\text {aq }}$ with increasing HP $\beta C D$ concentration (Fig. 8). As shown in Figure 9, the shrinking UWL with increasing HP $\beta C D$ concentration also causes the progesterone concentration at the surface of the membrane to increase and eventually become equal to the progesterone concentration in the bulk as the concentration of HP $\beta C D$ increases. In this way, complexation with cyclodextrins effectively shorts out the UWL by facilitating the transport of drug to the membrane surface such that $C_{\text {aq Membrane Surface }} \approx C_{\text {aq Bulk, and }}$ permeation across the intestinal membrane becomes rate limiting. ${ }^{22}$ At the same time, $P_{\mathrm{m}}$ decreases rapidly with increasing cyclodextrin concentration (Fig. 7). This may also be attributed to the high binding constant between progesterone and HP $\beta C D$, which causes the amount of free drug available for membrane permeation to decrease rapidly with increasing cyclodextrins concentration, as described in Eqs. (8) and (10). Figure 7 contains the overall predicted $P_{\text {eff }}$ as a function of cyclodextrins concentration in the rat intestinal perfusion model. The predicted $P_{\text {eff }}$ was calculated via Eq. (20). Excellent agreement was obtained between the experimental and predicted progesterone $P_{\text {eff }}$ values at all of the HP $\beta C D$ concentrations tested. The overall $P_{\text {eff }}$ stays relatively constant at very low $\mathrm{HP} \beta C D$ concentrations, but then decreases rapidly with increasing cyclodextrin concentration. This results because at very low cyclodextrin concentrations, the overall $P_{\text {eff }}$ is limited by the UWL. However, the rapidly decreasing effective $h_{\text {aq }}$ with increasing HP $\beta C D$ concentration causes $P_{\text {aq }}$ to markedly increase, such the UWL is quickly eliminated and the overall $P_{\text {eff }}$ becomes membrane controlled at higher $\mathrm{HP} \beta \mathrm{CD}$ concentrations. This is evidenced by the fact that the experimental and predicted values of $P_{\text {eff }}$ are essentially equal to the predicted values of $P_{\mathrm{m}}$ at the higher HP $\beta C D$ concentrations of 0.25 and $2.5 \mathrm{mM}$ (Fig. 7). It is acknowledged that validation of any mathematical model is a continuous process, and additional data would be of benefit.

It should be emphasized that the shrinking effective $h_{\mathrm{aq}}$ results directly from the decrease in free fraction of drug with increasing cyclodextrins concentration. Indeed, the UWL thickness experienced by the free drug remains constant with increasing cyclodextrins concentration. However, since the free fraction of drug decreases with increasing cyclodextrin concentration and it is assumed that the drug-cyclodextrin complex does not experience a UWL thickness (i.e., $h_{\mathrm{aq}(B)}=0$ ), the overall effective UWL thickness decreased as bound fraction increases and free fraction decreases (i.e., $\left.h_{\mathrm{aq}}=F h_{\mathrm{aq}(F)}\right)$. As stated in the model assumptions, this model does not consider cases in which interaction between the free cyclodextrin and the membrane exists.

\section{CONCLUSIONS}

In conclusion, this work demonstrates that when using cyclodextrins as pharmaceutical solubilizers, a trade-off exists between solubility increase and permeability decrease that governs the overall fraction of drug absorbed. Given these opposing effects, both apparent solubility and permeability considerations must be taken into account, to strike the appropriate balance in order to achieve optimal absorption from a cyclodextrin-based formulation. The quasi-equilibrium mass transport analysis developed in this work could successfully predict the $P_{\text {eff }}$ dependence on HP $\beta C D$ concentration with knowledge of $K_{11 \mathrm{aq}}, P_{\mathrm{m}(\mathrm{F})}, P_{\mathrm{aq}(F)}$ and the aqueous diffusion coefficients of the free drug and cyclodextrins, $D_{\mathrm{aq}(F)}$ 
and $D_{\text {aq(CD) }}$. Knowledge of the solubility-permeability interplay and the dependence of these parameters on cyclodextrin concentration enables the more efficient and intelligent use of cyclodextrins in oral drug product formulation development.

\section{REFERENCES}

1. Dahan A, Hoffman A. 2008. Rationalizing the selection of oral lipid based drug delivery systems by an in vitro dynamic lipolysis model for improved oral bioavailability of poorly water soluble drugs. J Control Release 129:1-10.

2. Lipinski CA, Lombardo F, Dominy BW, Feeney PJ. 2001. Experimental and computational approaches to estimate solubility and permeability in drug discovery and development settings. Adv Drug Deliv Rev 46:3-26.

3. Van de Waterbeemd H, Smith DA, Beaumont K, Walker DK. 2001. Property-based design: Optimization of drug absorption and pharmacokinetics. J Med Chem 44:1313-1333.

4. Dahan A, Amidon GL. 2008. Gastrointestinal dissolution and absorption of class II drugs. In: Van de Waterbeemd H, Testa B, editors. Drug bioavailability: Estimation of solubility, permeability, absorption and bioavailability, 2nd edition. Weinheim, Germany: Wiley-VCH. pp 33-51.

5. Dahan A, Hoffman A. 2006. Enhanced gastrointestinal absorption of lipophilic drugs. In: Touitou E, Barry BW, editors. Enhancement in drug delivery, Boca Raton, FL: CRC Press. pp 111-127.

6. Brewster ME, Loftsson T. 2007. Cyclodextrins as pharmaceutical solubilizers. Adv Drug Deliv Rev 59:645-666.

7. Davis ME, Brewster ME. 2004. Cyclodextrin-based pharmaceutics: Past, present and future. Nat Rev Drug Discov 3:1023.

8. Loftsson T, Brewster ME. 1996. Pharmaceutical applications of cyclodextrins. 1. Drug solubilization and stabilization. J Pharm Sci 85:1017-1025.

9. Rajewski RA, Stella VJ. 1996. Pharmaceutical applications of cyclodextrins. 2. In vivo drug delivery. J Pharm Sci 85:11421169 .

10. Amidon GL, Lennernas H, Shah VP, Crison JR. 1995. A theoretical basis for a biopharmaceutic drug classification: The correlation of in vitro drug product dissolution and in vivo bioavailability. Pharm Res 12:413.

11. Lobenberg R, Amidon GL. 2000. Modern bioavailability, bioequivalence and biopharmaceutics classification system. New scientific approaches to international regulatory standards. Eur J Pharm Biopharm 50:3-312.

12. Martinez MN, Amidon GL. 2002. A mechanistic approach to understanding the factors affecting drug absorption: A review of fundamentals. J Clin Pharmacol 42:620-643.

13. Yu LX, Amidon GL, Polli JE, Zhao H, Mehta MU, Conner DP, Shah VP, Lesko LJ, Chen ML, Lee VHL, Hussain AS. 2002. Biopharmaceutics classification system: The scientific basis for biowaiver extensions. Pharm Res 19:921.

14. Carrier RL, Miller LA, Ahmed I. 2007. The utility of cyclodextrins for enhancing oral bioavailability. J Control Release 123: 78-799.

15. Loftsson T, Jarho P, Masson M, Jarvinen T. 2005. Cyclodextrins in drug delivery. Expert Opin Drug Deliv 2:335-351.

16. Loftsson T, Vogensen SB, Brewster ME, Konrádsdóttir F. 2007. Effects of cyclodextrins on drug delivery through biological membranes. J Pharm Sci 96:2532-2546.

17. Loftsson T, Brewster ME, Masson M. 2004. Role of cyclodextrins in improving oral drug delivery. Am J Drug Deliv 2:261.

18. Rao VM, Stella VJ. 2003. When can cyclodextrins be considered for solubilization purposes? J Pharm Sci 92:927-932.
19. Miller LA, Carrier RL, Ahmed I. 2007. Practical considerations in development of solid dosage forms that contain cyclodextrin. J Pharm Sci 96:1691-1707.

20. Dahan A, Hoffman A. 2006. Use of a dynamic in vitro lipolysis model to rationalize oral formulation development for poor water soluble drugs: Correlation with in vivo data and the relationship to intra-enterocyte processes in rats. Pharm Res 23:2165-2174.

21. Higuchi T. 1960. Physical chemical analysis of percutaneous absorption process from creams and ointments. J Soc Cosmet Chem 11:85-97.

22. Amidon GE, Higuchi W, Ho N. 1982. Theoretical and experimental studies of transport of micelle-solubilized solutes. J Pharm Sci 71:77-84.

23. Higuchi T, Connors KA. 1965. Phase-solubility techniques. Adv Anal Chem Instrum 4:117-212.

24. Dahan A, Amidon GL. 2009. Grapefruit juice and its constituents augment colchicine intestinal absorption: Potential hazardous interaction and the role of P-glycoprotein. Pharm Res 26:883-892.

25. Dahan A, Amidon GL. 2009. Segmental dependent transport of low permeability compounds along the small intestine due to $\mathrm{P}$ glycoprotein: The role of efflux transport in the oral absorption of BCS class III drugs. Mol Pharm 6:19-28.

26. Miller JM, Dahan A, Gupta D, Varghese S, Amidon GL. 2009 Quasi-equilibrium analysis of the ion-pair mediated membrane transport of low-permeability drugs. J Control Release 137:3137.

27. Wohnsland F, Faller B. 2001. High-throughput permeability $\mathrm{pH}$ profile and high-throughput alkane/water log $\mathrm{P}$ with artificial membranes. J Med Chem 44:923-930.

28. Dahan A, Amidon GL. 2009. Small intestinal efflux mediated by MRP2 and BCRP shifts sulfasalazine intestinal permeability from high to low, enabling its colonic targeting. Am J Physiol Gastrointest Liver Physiol 297:G371-G377.

29. Kim JS, Mitchell S, Kijek P, Tsume Y, Hilfinger J, Amidon GL. 2006. The suitability of an in situ perfusion model for permeability determinations: Utility for BCS class I biowaiver requests. Mol Pharm 3:686-694.

30. Fagerholm U, Johansson M, Lennernas H. 1996. Comparison between permeability coefficients in rat and human jejunum. Pharm Res 13:1336-1342.

31. Brewster ME, Noppe M, Peeters J, Loftsson T. 2007. Effect of the unstirred water layer on permeability enhancement by hydrophilic cyclodextrins. Int J Pharm 342:250-253.

32. Loftsson T, Konrádsdóttir F, Másson M. 2006. Influence of aqueous diffusion layer on passive drug diffusion from aqueous cyclodextrin solutions through biological membranes. Pharmazie 61:83-89.

33. Ribeiro ACF, Valente AJM, Santos CIAV, Prazeres MRA, Lobo VMM, Burrows HD, Esteso MA, Cabral AMTDPV, Veiga FJB. 2007. Binary mutual diffusion coefficients of aqueous solutions of $\alpha$-cyclodextrin, 2-hydroxypropyl-a-cyclodextrin, and 2hydroxypropyl- $\beta$-cyclodextrin at temperatures from $(298.15$ to 312.15) K. J Chem Eng Data 52:586-590.

34. Komiya I, Park J, Kamani A, Ho N, Higuchi W. 1980. Quantitative mechanistic studies in simultaneous fluid flow and intestinal absorption using steroids as model solutes. Int J Pharm 4:249-262.

35. Johnson D, Amidon G. 1988. Determination of intrinsic membrane transport parameters from perfused intestine experiments: A boundary layer approach to estimating the aqueous and unbiased membrane permeabilities. J Theor Biol 131:93106.

36. Kou J, Fleisher D, Amidon G. 1991. Calculation of the aqueous diffusion layer resistance for absorption in a tube: Application to intestinal membrane permeability determination. Pharm Res 8:298-305. 